\title{
Stabilization of femtosecond laser frequency combs with subhertz residual linewidths
}

\author{
A. Bartels, C. W. Oates, L. Hollberg, and S. A. Diddams \\ Time and Frequency Division, National Institute of Standards and Technology, 325 Broadway, Boulder, Colorado 80305
}

\begin{abstract}
We demonstrate that femtosecond laser frequency combs (FLFCs) can have a subhertz linewidth across their entire emission spectra when they are phase locked to a reference laser with a similarly narrow linewidth. Correspondingly, the coherence time of the comb components relative to the reference laser can be of the order of a few seconds. Thus we are able to detect high-contrast spectral interferograms at up to 10 -s integration time between two FLFCs locked to a common optical reference.
\end{abstract}

The development of visible continuous-wave (cw) lasers with subhertz linewidths has been essential on the way toward optical atomic frequency standards with projected fractional frequency uncertainties of $\approx 1 \times 10^{18}, 1-3$ When locked to a narrow atomic clock transition, these lasers serve as the local oscillator of the frequency standard and provide stable optical frequency output. A means to transfer the low-noise properties of the optical standard to other optical frequencies (or to the microwave domain) is essential for applications, such as optical frequency metrology and comparisons, spectroscopy, or generation of highly stable microwave clock signals. This task can be conveniently solved with a femtosecond laser frequency comb (FLFC) that operates as an extremely broadband optical frequency synthesizer. By comparing two independent FLFCs referenced to a common cw laser, it has been shown that such systems are capable of synthesizing optical and microwave frequencies with instabilities at or below a few parts in $10^{15}$ in $1 \mathrm{~s}$ of averaging, ${ }^{4,5}$ Although these experiments have yielded upper limits on how a FLFC might compromise the stability of a reference laser, the linewidth of the FLFC relative to the reference laser has not yet been fully explored. In related work, Shelton et al. ${ }^{6}$ observed millisecond relative coherence times and Kobayashi et al. $^{7}$ observed kilohertz linewidths between two FLFCs locked to each other. We recently investigated a case in which a FLFC is locked to one low-noise optical reference oscillator and beat against a second reference offset by $\approx 100 \mathrm{~nm}$ from the first. This established an upper limit on the linewidth of the FLFC components of $150 \mathrm{~Hz}$, limited by noise in the fiber delivery of the reference lasers. ${ }^{8}$ In this Letter we directly address the question of what the linewidth of a FLFC can be relative to a low-noise optical reference by comparing two systems that share a common reference. In doing so, we assess our ability to transfer the coherence of optical frequency standards with a subhertz linewidth to a multitude of optical frequencies spread out across hundreds of terahertz.

We employ two femtosecond lasers (NIST-BB1 and NIST-BB2, referred to with indices $i=1,2$ ) that emit a broadband continuum with spectral coverage from approximately 580 to $1080 \mathrm{~nm}$ at a $1-\mathrm{GHz}$ repetition rate. ${ }^{9}$ They are phase locked to a common cavity-stabilized diode laser at $657-\mathrm{nm}$ wavelength (or a frequency $f_{\mathrm{LD}}=456 \mathrm{THz}$ ). ${ }^{5,10}$ To achieve this, we phase lock heterodyne beat signals at frequencies $f_{b, i}$ between $f_{\mathrm{LD}}$ and the neighboring component of both FLFCs (with mode numbers $n_{i} \approx 456,000$ ) to a stable radio-frequency $(\mathrm{RF})$ source. Also, the carrier-envelope offset frequencies $f_{0, i}$ of the lasers are phase locked to a second RF source by use of a $2 f-3 f$ self-referencing technique ${ }^{10}$ such that the repetition rates are given by $f_{R, i}=\left(\begin{array}{lll}f_{\mathrm{LD}} & f_{b, i} & f_{0, i}\end{array}\right) / n_{i}$ and the frequency comb components are $f_{k_{i}, i}=$ $f_{\mathrm{LD}}+k_{i} / n_{i} \times\left(f_{\mathrm{LD}} \quad f_{b, i} \quad f_{0, i}\right)$, with $k_{i}=$ $0, \pm 1, \pm 2, \ldots$. Subsequently, we set the repetition rates, i.e., the comb spacings, to be equal by choosing $n_{1}=n_{2}$ and $f_{0,1}+f_{b, 1}=f_{0,2}+f_{b, 2}$. With this scheme the residual noise on the diode laser largely cancels out in the following intercomparison experiments, as we can see by subtracting the two equations above. This yields an upper limit for the residual (i.e., intrinsic or technical noise in the two FLFCs that is not suppressed by the servo systems) phase noise that determines the linewidth of the FLFCs.

The average linewidth of a group of comb components is measured around $900-\mathrm{nm}$ wavelength $\left(\approx 250 \mathrm{~nm}\right.$ from $\left.f_{\mathrm{LD}}\right)$ by detection of a heterodyne beat signal between the FLFCs after a filter that transmits the infrared portions of the lasers. This beat appears at frequency $\Delta f_{0}=f_{0,1} \quad f_{0,2}$ when the pulses from both lasers are temporally overlapped on a Si p-i-n photodiode. The relative pulse delay is adjusted electronically before the measurement by momentarily detuning one of the phase locks to slightly vary the repetition rate of one of the FLFCs. Figure 1(a) shows the RF spectrum of this optical beat taken with $1-\mathrm{kHz}$ resolution bandwidth (RBW). It shows a narrow, RBW-limited peak at the carrier frequency with a broad pedestal that contains uncompensated amplitude and phase noise present on both lasers. The central peak (taken within four times the RBW) contains $74 \%$ of the power in a $1-\mathrm{MHz}$ span. The spectrum in the inset of Fig. 1(a) was taken with $1-\mathrm{Hz}$ RBW and again shows a RBW-limited carrier together with a number of distinct lines that result from mechanical resonances of the setup. Higher-resolution 

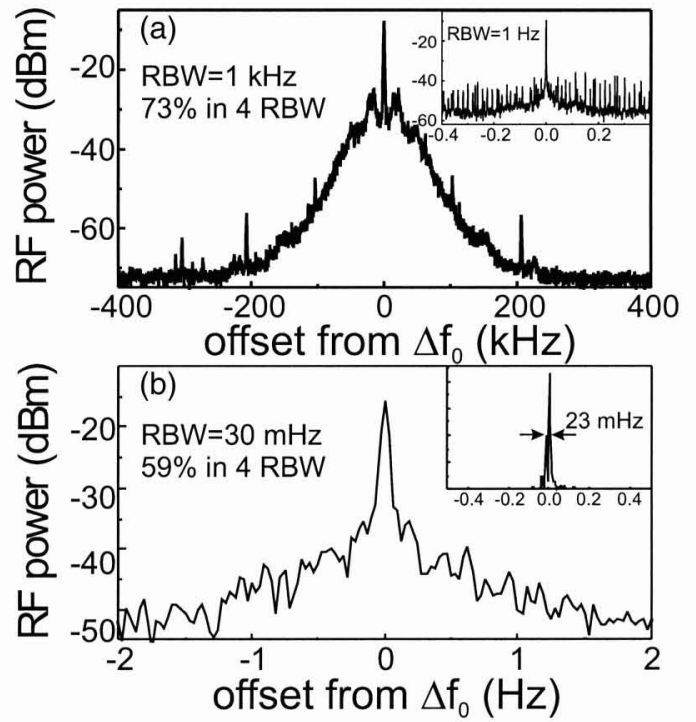

Fig. 1. Heterodyne beat measured around $900 \mathrm{~nm}$ between the two FLFCs with $\Delta f_{0}=101 \mathrm{MHz}$. (a) Measurement with a RF spectrum analyzer at $1-\mathrm{kHz} \mathrm{RBW}$. Inset, measurement with $1-\mathrm{Hz} \mathrm{RBW}$. (b) Measurement of the beat mixed to $10 \mathrm{~Hz}$ with a fast Fourier transform spectrum analyzer at $30-\mathrm{mHz} \mathrm{RBW}$ (inset, with $3-\mathrm{mHz} \mathrm{RBW}$, linear scale). The fraction of overall power contained in the central peaks is indicated.

spectra were obtained by mixing the $\Delta f_{0}$ beat with a synthesizer offset by $10 \mathrm{~Hz}$ and recording the downconverted signal with a fast Fourier-transform spectrum analyzer. The result taken with a RBW of $30 \mathrm{mHz}$ is shown in Fig. 1(b), where the fractional power contained in the RBW-limited central peak is still $59 \%$ of the overall power. With the RBW reduced to $3 \mathrm{mHz}$, we see a linewidth of approximately $20 \mathrm{mHz}$.

When the power contained in the noise pedestal of the heterodyne beat is not negligible compared with that in the carrier, the $3-\mathrm{dB}$ linewidth is not a good indication of the coherence time of the FLFCs. A more rigorous determination comes from a phase-noise measurement. The $\Delta f_{0}$ beat is mixed in quadrature to dc with a low-noise RF source. The voltage fluctuations from the mixer are recorded with a fast Fouriertransform spectrum analyzer, yielding the phase-noise spectrum $S_{\phi}(f)$ as shown in Fig. 2. This spectrum shows $1 / f^{2}$ behavior up to $10 \mathrm{~Hz}$ before a multitude of acoustic and vibrational resonances at up to a few tens of kilohertz appear. The peaks around 50 and $100 \mathrm{kHz}$ contain the resonances of our feedback loops. The integrated phase noise $\phi_{\text {int. }}(f)=\int_{f}^{1 \mathrm{MHz}} S_{\phi}\left(f^{\prime}\right) \mathrm{d} f^{\prime}$ (see Fig. 2) reaches $1 \mathrm{rad}$ at $f=0.2 \mathrm{~Hz}$, from which we infer a coherence time of $5 \mathrm{~s}$. It is clear in our experiments that the present limitation to the measured coherence time is not of fundamental nature but is due to mechanical vibrations and air currents in our setup.

To confirm the high mutual coherence between the FLFCs, we measured spectral interferograms (SIs) between them at wavelengths around 850 and $670 \mathrm{~nm} .{ }^{11}$ This measurement requires that the combs are coincident in frequency $\left(n_{1}=n_{2}, f_{0,1}=f_{0,2}\right.$ and $\left.f_{b, 1}=f_{b, 2}\right)$ and overlapped in time. The signal as a function of the angular light frequency $\omega$ is then given as

$$
\begin{aligned}
I(\omega)= & I_{1}(\omega)+I_{2}(\omega)+2 C\left[I_{1}(\omega) I_{2}(\omega)\right]^{1 / 2} \\
& \times \cos (\omega \tau+\alpha),
\end{aligned}
$$

where $I_{i}(\omega)$ are the spectra of the individual lasers and $\tau$ is the temporal delay between the pulses as they are dispersed by a grating spectrometer and illuminate a CCD array. $\alpha$ is a constant phase, and $C$ is a contrast factor that will be discussed below. Mode matching of the beams from both lasers is achieved with a single-mode fiber. The polarizations are projected on a common axis with a polarizer in the combined beam. Figure 3(a) shows a SI recorded on a CCD array around $850 \mathrm{~nm}$ with an integration time of $T_{\text {int. }}=1 \mathrm{~s}$. The contrast of the SI can be determined by inserting the measured individual laser spectra $I_{i}(\omega)$ (gray curves in Fig. 3) and $\tau$ (extracted from the fringe spacing of the experimental SI) into Eq. (1) and adjusting $C$ and $\alpha$ to match the measured SI. Using this procedure, we find an experimental contrast of $C_{\text {exp. }}=65 \%$ for the data of Fig. 3(a). Figure 3(b) shows a SI near $850 \mathrm{~nm}$ with $T_{\text {int. }}=10 \mathrm{~s}$. Although the contrast has decreased to $C_{\text {exp. }}=45 \%$, interference fringes are still clearly visible.

Two main factors reduce the contrast of the SIs from the ideal 100\%: the phase noise of the FLFCs and the spectrometer resolution. These effects can be accounted for by a theoretical contrast factor:

$$
C_{\text {th. }}(\omega)=\frac{\sin \left[\phi_{\text {int. }}^{\prime}\left(T_{\text {int. }}^{1}, \omega\right)\right]}{\phi_{\text {int. }}^{\prime}\left(T_{\text {int. }}^{1}, \omega\right)} \frac{\sin (\pi R \tau)}{\pi R \tau} .
$$

Here, $\phi_{\text {int. }}^{\prime}\left(T_{\text {int. }}^{1}, \omega\right)$ is the measured integrated phase noise $\phi_{\text {int. }}(f)$ at frequency $f=T_{\text {int. }}^{1}$ scaled by a factor $\omega /(2 \pi \times 333 \mathrm{THz})$ to account for the fact that $\phi_{\text {int. }}(f)$ has been measured at $\approx 900 \mathrm{~nm}(333 \mathrm{THz}) . \quad R$ is the measured spectrometer resolution of $\approx 750 \mathrm{GHz}$. Note that $C_{\mathrm{th}}$. can be negative if the arguments of the sine functions in Eq. (2) become greater than $\pi$. However, if this is the case, $\left|C_{\text {th. }}\right|$ can never be greater than $\approx 22 \%$, excluding our experimental conditions from this regime. We now use the individual spectra of both FLFCs and $C_{\text {th. }}$ to construct the theoretically expected SIs according to Eq. (1), which are shown as dashed curves in Fig. 3. For the SIs taken around $850 \mathrm{~nm}, C_{\text {th. }}$ is $\approx 75 \%$ for $T_{\text {int. }}=1 \mathrm{~s}$ and $\approx 47 \%$ for $T_{\text {int. }}=10 \mathrm{~s}$. The $C_{\text {th. }}$ and the calculated SIs are in

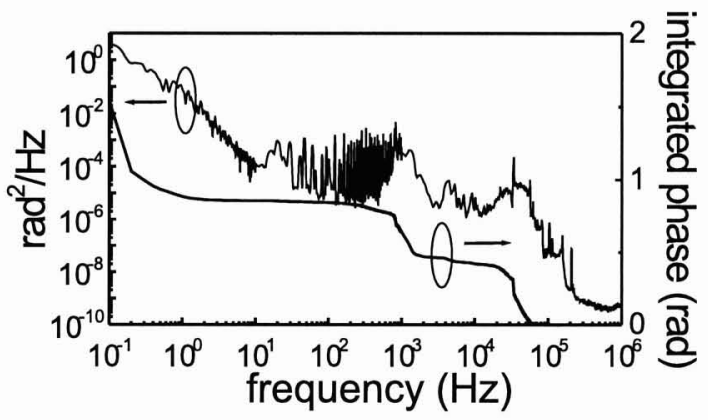

Fig. 2. Left-hand axis, phase-noise power spectral density of the $\Delta f_{0}$ beat, equivalent to the phase noise of the optical frequency comb components around $900 \mathrm{~nm}(333 \mathrm{THz})$. Right-hand axis, integrated phase noise. 


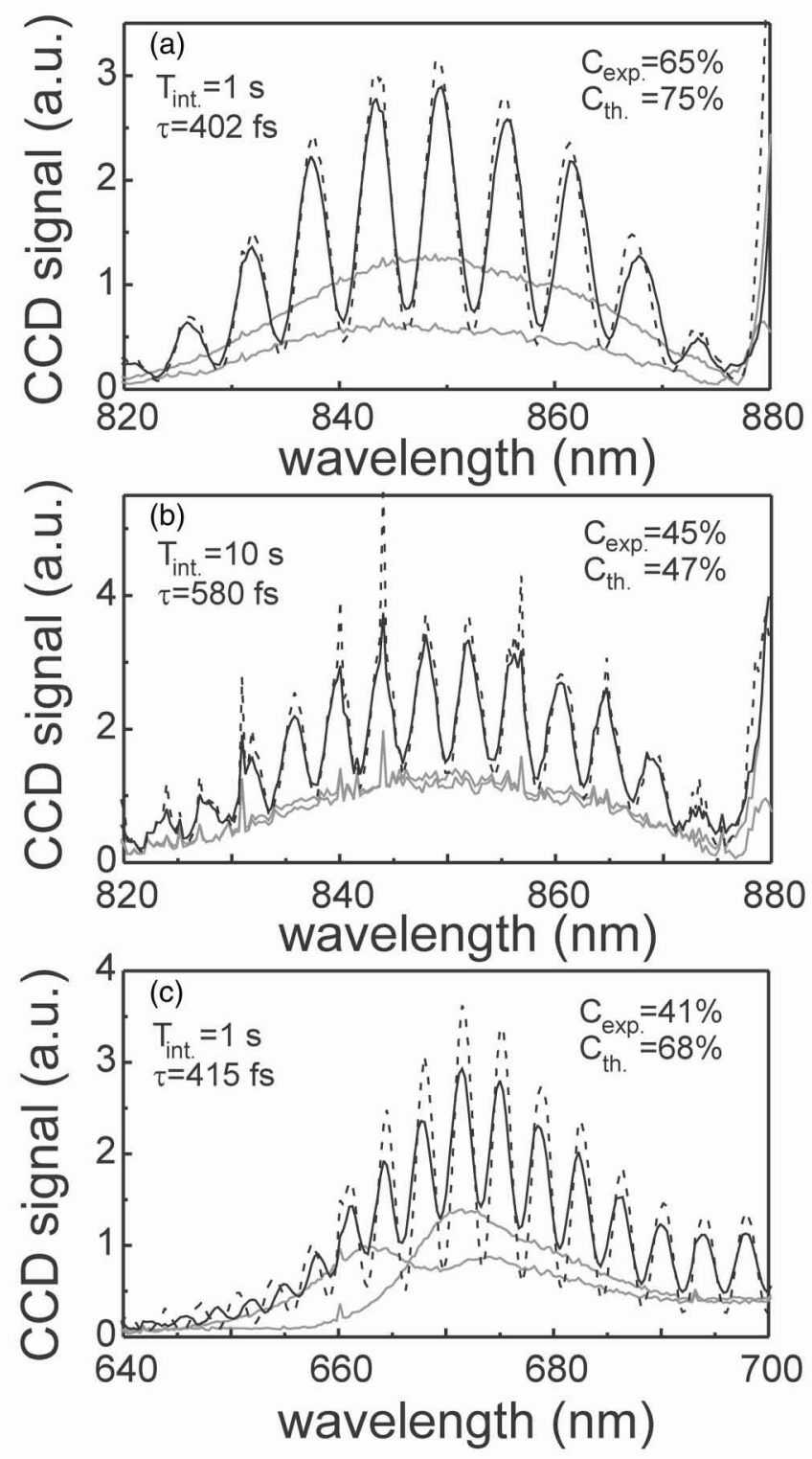

Fig. 3. SIs (solid black curves) between NIST-BB1 and NIST-BB2, the individual laser spectra (solid gray curves), and theoretical SIs calculated from the individual spectra (dashed curves): (a) using the infrared output of the FLFCs and $T_{\text {int. }}=1 \mathrm{~s}$, (b) same as (a) with $T_{\text {int. }}=10 \mathrm{~s}$, (c) using the visible output and $T_{\text {int. }}=1 \mathrm{~s}$. The pulse spacing $\tau$ and the experimental and theoretical contrast are also indicated.

good agreement with the experiment. These results demonstrate that the independent and complementary measurements of phase-noise $S_{\phi}(f)$ and spectral interferograms yield excellent agreement. We also measured a SI with $T_{\text {int. }}=1 \mathrm{~s}$ close to the reference wavelength around $670 \mathrm{~nm}$ [see Fig. 3(c)], yielding $C_{\text {th. }}=68 \%$ and $C_{\text {exp. }}=41 \%$. The difference between $C_{\text {th. }}$ and $C_{\text {exp. }}$ in this case is likely the result of differences in the mechanical setup at $670 \mathrm{~nm}$ and that at $850 \mathrm{~nm}$, with which $\phi_{\text {int. }}(f)$ was measured.

From the viewpoint of synthesizing highly coherent light, it is important to point out that the use of an optical reference is advantageous compared with using a microwave reference. ${ }^{12}$ A microwave reference needs to be multiplied by a factor of $\approx 10^{5}$ to reach visible frequencies, whereas an optical reference requires only a factor of $\approx 1\left(k_{i} / n_{i}\right)$. This greatly reduces the multiplicative factor by which phase noise in the control loops enters the FLFC output. This difference is important for our improvement of relative coherence by at least 3 orders of magnitude over earlier experiments in which two FLFCs were locked to each other in the microwave domain. ${ }^{6}$

In conclusion, we have demonstrated that the components of a femtosecond laser frequency comb can have a linewidth of the order of $20 \mathrm{mHz}$ relative to a low-noise reference laser. The phase noise of the FLFC components has a relative coherence time of $5 \mathrm{~s}$, limited by the measurement setup. This is confirmed by the visibility of high-contrast spectral interferograms between the FLFCs at integration times of up to $10 \mathrm{~s}$. Calculated interferograms that account for the independently measured phase noise on $\Delta f_{0}$ agree well with the measurements. The absence of degradation of fringe contrast as one moves from the reference wavelength of 657 to $850 \mathrm{~nm}$ proves that the FLFCs are capable of transferring an extremely narrow and coherent input signal over their entire emission bandwidth.

We thank R. W. Fox and J. C. Bergquist for their contributions to this experiment. This work was funded in part by NASA. A. Bartels's e-mail address is albrecht@boulder.nist.gov.

Note added in proof: We recently compared the reference laser used in this experiment and a second low-noise $\mathrm{cw}$ laser separated by $76 \mathrm{THz}$ via NIST-BB1 and established an upper limit of $\approx 4 \mathrm{~Hz}$ on the absolute linewidth of the individual components of our FLFCs as well as on our reference laser.

\section{References}

1. Ch. Salomon, D. Hils, and J. L. Hall, J. Opt. Soc. Am. B 5, 1576 (1988).

2. B. C. Young, F. C. Cruz, J. C. Bergquist, and W. M. Itano, Phys. Rev. Lett. 82, 3799 (1999).

3. R. J. Rafac, B. C. Young, J. A. Beall, W. M. Itano, D. J. Wineland, and J. C. Bergquist, Phys. Rev. Lett. 85, $2463(2000)$.

4. S. A. Diddams, L. Hollberg, L.-S. Ma, and L. Robertsson, Opt. Lett. 27, 58 (2002).

5. A. Bartels, S. A. Diddams, T. M. Ramond, and J. Hollberg, Opt. Lett. 28, 663 (2003).

6. R. K. Shelton, L.-S. Ma, H. C. Kapteyn, M. M. Murnane, J. L. Hall, and J. Ye, Science 293, 1286 (2001).

7. Y. Kobayashi, T. Torizuka, and Z. Wei, Opt. Lett. 28, 746 (2003).

8. S. A. Diddams, A. Bartels, T. M. Ramond, C. W. Oates, S. Bize, E. A. Curtis, J. C. Bergquist, and L. Hollberg, IEEE J. Sel. Top. Quantum Electron. 9, 1072 (2003).

9. A. Bartels and H. Kurz, Opt. Lett. 27, 1839 (2002).

10. T. M. Ramond, S. A. Diddams, L. Hollberg, and A. Bartels, Opt. Lett. 27, 1842 (2002).

11. L. Lepetit, G. Cheriaux, and M. Joffre, J. Opt. Soc. Am. B 12, 2467 (1995) and references therein.

12. J. Ye, J. L. Hall, and S. A. Diddams, Opt. Lett. 25, 1675 (2000). 